\title{
Effects of high-frequency repetitive transcranial magnetic stimulation of primary motor cortex on laser-evoked potentials in migraine
}

\author{
Marina de Tommaso • Filippo Brighina $\cdot$ Brigida Fierro $\cdot$ Vito Devito Francesco \\ Roberto Santostasi • Vittorio Sciruicchio - Eleonora Vecchio • Claudia Serpino • \\ Paolo Lamberti • Paolo Livrea
}

Received: 26 May 2010/ Accepted: 27 July 2010/Published online: 17 August 2010

(C) Springer-Verlag 2010

\begin{abstract}
The aim of this study was to examine the effects of high-frequency (HF) repetitive transcranial magnetic stimulation (rTMS) of the left primary motor cortex (M1) on subjective pain and evoked responses induced by laser stimulation (LEPs) of the contralateral hand and supraorbital zone in a cohort of migraine patients without aura during the inter-critical phase, and to compare the effects with those of non-migraine healthy controls. Thirteen migraine patients and 12 sex- and age-matched controls were evaluated. Each rTMS session consisted of 1,800 stimuli at a frequency of $5 \mathrm{~Hz}$ and $90 \%$ motor threshold intensity. Sham (control) rTMS was performed at the same stimulation position. The vertex LEP amplitude was reduced at the trigeminal and hand levels in the shamplacebo condition and after rTMS to a greater extent in the migraine patients than in healthy controls, while the laser pain rating was unaffected. These results suggest that HF rTMS of motor cortex and the sham procedure can both modulate pain-related evoked responses in migraine patients.
\end{abstract}

Keywords Migraine - Laser-evoked potentials .

Motor cortex $\cdot$ Repetitive transcranial magnetic stimulation

M. de Tommaso $(\varangle)$. V. D. Francesco - R. Santostasi

V. Sciruicchio - E. Vecchio - C. Serpino - P. Lamberti .

P. Livrea

Neurophysiopathology of Pain Unit,

Neurological and Psychiatric Sciences Department,

Neurological Clinic, Policlinico, University of Bari

“Aldo Moro", Piazza Giulio Cesare 11, 70124 Bari, Italy

e-mail:m.detommaso@neurol.uniba.it

F. Brighina - B. Fierro

Clinical Neuroscience Department,

University of Palermo, Palermo, Italy

\section{Introduction}

Transcranial magnetic stimulation (TMS) was first described by Barker et al. [1] as a non-invasive, painless way to stimulate the human brain. TMS has been applied to many neurological and psychiatric disorders to explore the pathophysiology of these conditions, the clinical diagnostic utility of different TMS techniques and their use as a possible treatment $[2,3]$. TMS is based on a time-varying magnetic field that generates an electric current inside the skull, which can be focused and restricted to small brain areas by appropriate stimulation coil geometry and size [4]. This current, if applied repetitively [repetitive TMS (rTMS)], induces cortical modulation that lasts beyond the time of stimulation [2]. The effect of this cortical modulation depends on the frequency of the stimulation used: increased and decreased excitability result from lowfrequency (LF) and high-frequency (HF) TMS, respectively. RTMS can thus guide brain plasticity and consequently can be used to treat chronic pain, a disorder that is associated with substantial reorganization of CNS activity [2].

The use of rTMS in the primary motor cortex (M1) to control pain was first reported by Migita et al. [5], who showed pain reduction in two patients treated by LF $(<0.2 \mathrm{~Hz})$ rTMS. Since then, growing evidence has supported the potential beneficial effects of motor cortex rTMS for pain control in chronic pain patients [6, 7]. However, many questions remain to be addressed before any firm conclusions about this therapy can be drawn [2]. Several studies attempted to explore the mechanisms of M1 interaction for pain control using objective methods, including laser-evoked potentials, which are a reliable measure of nociceptive pathways function [8]. In normal subjects, LF TMS of the motor cortex reduced subjective pain and cortical activation induced by intradermal 
injection of capsaicin [9], but increased evoked responses and subjective pain related to a-delta fibers activation by laser stimuli [10]. HF TMS of the motor cortex increased the cold pain threshold [11] and tolerance of painful mechanical stimuli [12] in normal study participants. Moreover, the voluntary movement preparation inhibits subjective pain and evoked responses induced by laser stimuli, thus confirming that activation of the motor cortex can reduce the nociceptive cortical recruitment induced by acute painful stimulation [13]. In chronic neuropathic pain patients, the activation of the motor cortex by HF rTMS seems to alleviate painful symptoms, probably through the restoration of GABAergic inhibition in the nociceptive cortex [14]. Accordingly, HF rTMS of M1 reduced vertex LEPs and laser pain in a group of chronic neuropathic pain patients [15].

Migraine is an incapacitating disorder of neurovascular origin, and prophylactic treatments are often inadequate to prevent it from becoming chronic. In some instances, HF rTMS alleviates migraine and psychogenic headaches that accompany major depression disorders [16]. In addition, dorsolateral prefrontal cortex (DLPFC) modulation has a positive effect in chronic migraine patients [17]. However, the altered cortical excitability that characterizes migraine can make the effects of rTMS unpredictable [3]. Given that in migraine patients LEP pattern seems to reflect the modality of nociceptive cortex activation as well as the effects of treatments [18], we aimed to examine the effects of HF rTMS of the primary motor cortex on subjective pain and evoked responses induced by laser stimulation of the contralateral hand and trigeminal zone in a cohort of migraine patients without aura during the inter-critical phase, and to compare the effects with those of nonmigraine, healthy controls.

\section{Methods}

\section{Subjects}

Twelve healthy, right-handed subjects (10 females) from 21 to 38 years of age (mean $32.2 \pm 2.9$ ) participated in the study. The migraine group consisted of 13 right-handed migraine patients without aura (11 females) ranging in age from 21 to 39 years (mean $32.6 \pm 4.1$ ) and that had been diagnosed according to the International Classification of Headache Disorders (ICHD II) criteria (cod. 1.1) [19]. Migraine patients were recorded during the inter-critical phase at least $72 \mathrm{~h}$ after the last attack and $48 \mathrm{~h}$ before the next one, as ascertained by a telephone interview. Exclusion criteria were as follows: preventive treatment for migraine or treatment with any drug which acts on the SNC in the previous 3 months, treatment with any analgesic drug in the pervious $72 \mathrm{~h}$, co-morbidity for general medical and neurological diseases, and any other psychiatric disease as described by the Diagnostic and Statistical Manual of Mental Disorders (DSM-IV) [20]. Patients and controls were similar in age [ANOVA $F=0.23$, non-significant (NS)] and education (mean number of years of education was $12.1 \pm 0.8$ and $12.8 \pm 0.5$ for patients and controls, respectively; ANOVA $F=0.12$, NS). The selected migraine patients reported a mean of $3.2 \pm 1.1$ days with headache per month over the previous 3 months.

\section{TMS}

All subjects were comfortably seated in a chair and instructed to be as relaxed as possible. The rTMS was delivered over the hand motor cortex of the left hemisphere through a water-cooled figure eight coil powered by a MagPro X 100 (MedTronic) magnetic stimulator. The stimulating coil was placed over the site that optimally elicited responses in the contralateral abductor pollicis brevis (ABP) target muscle (termed the APB hotspot). The motor threshold (MT) was measured as the minimum stimulus intensity that elicited a motor evoked potential (MEP) of at least $50 \mu \mathrm{V}$ in 5 or more of 10 consecutive stimulations in the right APB hot spot. To establish the motor threshold, electromyography (EMG) signals were recorded from the right APB muscle using 0.9- $\mathrm{cm}$ diameter $\mathrm{Ag}-\mathrm{AgCl}$ surface electrodes placed $3 \mathrm{~cm}$ apart over the center and tendon of the muscle. The EMG activity was recorded with a band-pass filter between 10 and $1,000 \mathrm{~Hz}$ and a display gain ranging from 50 to $200 \mu \mathrm{V} / \mathrm{cm}$. Each rTMS session consisted of 1,800 stimuli divided in 12 trains at a frequency of $5 \mathrm{~Hz}$ and $90 \%$ MT intensity, and stimuli were separated by a 10 -s pause. Sham (control) rTMS was performed at the same stimulation position with the coil tilted approximately $45^{\circ}$ over the scalp.

Laser-evoked potentials

During LEP recording, the subjects lay on a couch in a warm, semi-dark room and were awake and relaxed with their eyes open. Both the subject and the experimenters wore protective goggles or glasses during data acquisition. LEPs were obtained using surface recording electrodes placed at $\mathrm{Cz}$ and $\mathrm{Pz}$, with reference to the nasion, and at the $\mathrm{T} 3$ and $\mathrm{T} 4$ positions with reference to the $\mathrm{Fz}$ derivation (10-20 international system), by means of a MICROMED EEG apparatus (Micromed Brain Quick, Mogliano Veneto, Italy). An additional electrode was positioned above the right eyebrow for electro-oculogram (EOG) recording. The ground electrode was located at Fpz.

Cutaneous heat stimuli were delivered by a $\mathrm{CO}_{2}$ laser [wavelength $10.6 \mathrm{um}, 2 \mathrm{~mm}$ beam diameter (ELEN, 
Florence, Italy)] on the dorsum of the right hand and the right supraorbital zone. The duration of the stimulus was $25 \mathrm{~ms}$. The laser pain threshold (PTh) was established in the basal session by delivering a series of stimuli at increasing and decreasing intensities using $0.5 \mathrm{~W}$ steps. The pain threshold was the lowest intensity that enabled at least $50 \%$ of the stimuli to be perceived as a painful pinprick using a 10-point verbal analogical scale in which ' 0 ' corresponds to no sensation, ' 4 ' to the pain threshold and ' 10 ' to intolerable pain. The basal laser intensity was set at two steps over the pain threshold. In rTMS and sham sessions, the mean laser intensity was $8.1 \pm 0.2 \mathrm{~W}$ in controls and $7.9 \pm 0.3 \mathrm{~W}$ in migraine patients (ANOVA $F=0.23$, NS). To verify the modifications induced in the sham and the rTMS sessions, we asked all subjects to rate the laser pain on a $0-100$ visual analogical scale (VAS) at the end of a stimulation series, where ' 0 ' corresponds to no pain and ' 100 ' to the worst pain conceivable. In the basal, sham and rTMS sessions, the dorsum of the right hand and the right supraorbital zone was stimulated in random order by two consecutive series of 25 stimuli with an ISI of $10 \mathrm{~s}$ and an inter-series interval of $5 \mathrm{~min}$. An investigator blind to the clinical condition analyzed LEPs in $1 \mathrm{~s}$ intervals with $100 \mathrm{~ms}$ pre-stimulus time at a sampling rate of $512 \mathrm{~Hz}$ using advanced source analysis (ASA) software (version 4.6 by ANT). All runs containing transient responses exceeding $65 \mathrm{mV}$ in each recording channel were excluded from the average by an automatic artifact rejection algorithm. In addition, further artifacts were visually inspected, and an average of at least 15 artifact-free responses was obtained off-line for each stimulation series. For each stimulation site, an average across the two series of stimuli was obtained for the right hand and right supraorbital zone. LEPs were identified based on their latency and distribution, and three responses were labeled according to Valeriani et al. [21]. The N2a (namely N2) and P2 components were analyzed at the vertex $(\mathrm{CZ})$, and the N1 component was analyzed at the T3-Fz trace. The absolute latencies of the scalp potentials were measured at the highest peak of each response component, and the amplitude of each wave was measured from the baseline. The baseline was measured automatically by calculating the average signal of the whole sweep and subtracting it from the trace (ASA-vers. 4.6 by ANT software). In addition, the peak-to-peak amplitude was taken into consideration for the vertex biphasic LEP component (N2-P2).

Experimental procedures and statistical analysis

All subjects were informed of the experimental procedure and signed an informed consent document that was approved by the Bari Policlinico General Hospital Ethic Committee. After a basal LEP evaluation, each subject was submitted to the rTMS and sham sessions in a random sequence over two consecutive days. In the rTMS and sham sessions, LEPs were obtained immediately after the TMS modulation by stimulating the right hand and supraorbital zones in a random order using the procedure outlined above.

The one-way ANOVA was used to analyze the data where the LEP latency and amplitude and the laser pain rating were variables, the session (baseline, real rTMS and sham rTMS) within subject factor. To compare the variables across the three different sessions, a post hoc multiple comparison Bonferroni test was applied to single groups. In addition, we computed the percent variation of N2-P2 amplitude between the sham and the basal, the rTMS and the basal and the rTMS and the sham conditions, and then we compared these values between patients and controls, performing the Student's $t$ test, corrected for multiple comparisons.

\section{Results}

At both the hand and face levels, no significant difference were observed in the LEP latency across three sessions either in migraine patients or in control subjects (Table 1).

Table 1 Mean values \pm standard deviations (SD) of laser-evoked potentials latencies (LEP), expressed in $\mathrm{ms}$, in control subjects $(n=12)$ and migraine patients $(n=13)$

\begin{tabular}{|c|c|c|c|c|c|c|}
\hline \multirow[t]{2}{*}{ LEP latencies (ms) } & \multicolumn{2}{|l|}{ N1 } & \multicolumn{2}{|l|}{ N2 } & \multicolumn{2}{|l|}{ P2 } \\
\hline & Mean & SD & Mean & SD & Mean & SD \\
\hline \multicolumn{7}{|l|}{ Hand } \\
\hline \multicolumn{7}{|l|}{ Control subjects } \\
\hline Basal & 179.30 & 22.61 & 243.67 & 31.40 & 386.33 & 29.93 \\
\hline Sham & 179.33 & 34.06 & 234.90 & 37.88 & 358.40 & 36.32 \\
\hline rTMS & 178.28 & 27.62 & 234.00 & 36.85 & 352.00 & 46.44 \\
\hline \multicolumn{7}{|l|}{ Migraine patients } \\
\hline Basal & 162.90 & 21.50 & 231.83 & 22.42 & 348.00 & 49.23 \\
\hline Sham & 171.36 & 29.38 & 245.17 & 25.70 & 354.25 & 22.67 \\
\hline rTMS & 176.00 & 31.16 & 238.50 & 27.76 & 348.25 & 32.29 \\
\hline \multicolumn{7}{|l|}{ Face } \\
\hline \multicolumn{7}{|l|}{ Control subjects } \\
\hline Basal & 154.60 & 18.48 & 186.73 & 18.80 & 278.00 & 34.07 \\
\hline Sham & 160.71 & 25.32 & 185.38 & 22.66 & 289.00 & 28.53 \\
\hline rTMS & 146.53 & 9.05 & 188.38 & 39.55 & 288.00 & 38.95 \\
\hline \multicolumn{7}{|l|}{ Migraine patients } \\
\hline Basal & 140.18 & 43.27 & 168.36 & 59.79 & 277.64 & 25.03 \\
\hline Sham & 166.80 & 39.26 & 186.60 & 70.84 & 287.50 & 43.44 \\
\hline rTMS & 161.27 & 57.31 & 209.91 & 73.87 & 309.09 & 48.85 \\
\hline
\end{tabular}

The results of ANOVA for sessions and for the interaction diagnosis $x$ session were not significant 
In patients and controls, the laser pain rating showed a slight and non-significant reduction in rTMS and sham sessions with respect to the basal session, and this reduction was more evident at the face level (Fig. 1; Table 2). The N1 amplitude seemed not to be significantly modified by sham sessions or rTMS at the hand and trigeminal levels in patients and controls (Fig. 2a, b; Table 2). In migraine patients, both rTMS and sham sessions caused a slight N1 reduction with respect to the basal condition (Fig. 2a, b; Table 2). In patients and controls, the N2-P2 vertex complex appeared significantly modified across the different sessions at the hand level (Figs. 2a, 3; Table 2). In migraine patients, the sham rTMS caused a significant reduction in the vertex wave compared to the basal condition, but Bonferroni tests revealed that the real rTMS reduced more efficiently LEPs than sham treatment (Fig. 2a; Table 2). In control subjects, N2-P2 amplitude was significant reduced by rTMS compared to the basal condition, but no significant change was detectable between basal versus sham and sham versus rTMS conditions (Fig. 2a; Table 2). At the trigeminal level, ANOVA analysis revealed that the session factor caused a significant change in the N2-P2 amplitude, with a difference between the two groups (Fig. 2b; Table 2). In fact, while in control subjects, the LEP amplitude was slightly increased in the sham condition and reduced in the rTMS session, in the migraine patients the sham treatment provoked a significant reduction in the vertex complex, and this reduction was even greater following rTMS (Figs. 2b, 3). In migraine group, the reduction of N2-P2 amplitude prevailed with respect to controls, in rTMS versus basal condition at the hand level, and in sham versus rTMS, rTMS versus basal and rTMS versus sham condition at the face level (Fig. 4).

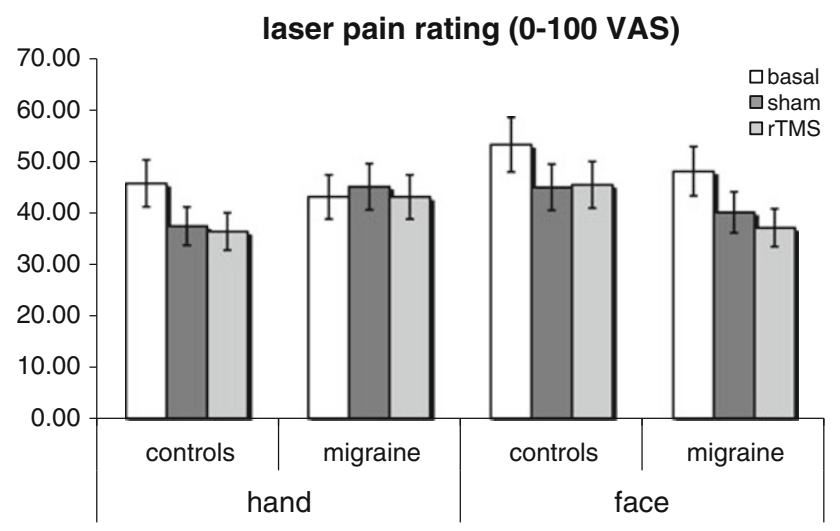

Fig. 1 Mean values and standard deviations of laser pain rating measured by score of $0-100$ VAS in control subjects $(n=12)$ and migraine patients without aura $(n=13)$ resulting from stimulation of the right hand and the right supraorbital zone in basal conditions and after sham TMS and $5 \mathrm{~Hz}$ rTMS exposure at the left primary motor cortex
The N2 and P2 amplitudes were also separately considered to examine the effects of sham and rTMS on the control and migraine groups, showing the same trend as the whole N2-P2 complex (Table 2). The Bonferroni test showed that in the control group, the P2 and the N2 obtained by the hand and the face stimulation were both reduced in rTMS condition, with respect to the basal situation (hand: $\mathrm{N} 2 p=0.049 ; \mathrm{P} 2 p=0.043$; face: $\mathrm{N} 2$ $p=0.49 ; \mathrm{P} 20.043)$, as well as in the migraine group (hand: $\mathrm{N} 2 p=0.03 ; \mathrm{P} 2 p=0.034$; face: $\mathrm{N} 2 p=0.024$; 2 $p=0.022$ ), where a significant effect of the sham rTMS was confirmed, with respect to real rTMS (hand: N2 $p=0.043 ; \quad \mathrm{P} 2 p=0.048 ;$ face: $\mathrm{N} 2 p=0.039, \quad \mathrm{P} 2$ $p=0.035$ ) and basal condition (hand: $\mathrm{N} 2 p=0.03$; $\mathrm{P} 2$ 0.032; face: $\mathrm{N} 2 p=0.036$; $\mathrm{P} 2 p=0.032$ ).

\section{Discussion}

This is the first study that has attempted to confirm the efficacy of motor cortex stimulation in reducing cortical responses to painful stimuli in migraine patients. HF rTMS of the motor cortex has been suggested for the treatment of other pain-related diseases since the initial report by Lefaucheur in 2001 [22]. The main results of our findings are that (1) sham and rTMS did not have a substantial effect on laser pain in patients and controls; (2) both sham and rTMS do not affect the N1 wave in either control or migraine group; (3) sham rTMS has a placebo effect on the vertex LEP amplitude in migraine patients; and (4) rTMS has an effect on the later LEP amplitudes in both control and migraine groups, with a more pronounced effect in the migraine patients.

In regard to point (1), laser pain was not significantly modified by motor cortex stimulation in normal subjects or migraine patients, despite a significant reduction of the evoked responses recorded from the vertex. Similarly, in a study on acute therapies for migraine attack, we observed no drug-induced effects on laser pain concomitant with the clear inhibition of laser-evoked responses [23]. This apparent contrast may be supported by the principle that LEPs reflect more the status of sensory pathways than the perception of subjective pain [8], despite a linear correlation between the intensity of pain perception and the amplitude of vertex LEPs is often present [24]. There are also evidences supporting a reducing effect of drugs with opioid activity (e.g. tramadol), on LEPs amplitude, without affecting laser pain perception [25].

Coming to the point (2), the M1 repetitive magnetic stimulation seemed to affect the LEP components differently, as the early temporal N1 component was substantially unmodified despite the slight reduction induced by the sham and the real stimulation procedure in migraine 
Table 2 Results of one-way ANOVA with conditions basal, sham and rTMS as factors $(d f=2)$ in migraine and control groups

\begin{tabular}{|c|c|c|c|c|c|c|c|c|}
\hline & VAS & N1 latency & N2 latency & P2 latency & N1 amplitude & N2 amplitude & P2 amplitude & N2-P2 amplitude \\
\hline \multicolumn{9}{|c|}{ Migraine patients $(n=12)$} \\
\hline Hand & $F=1.27$ & $F=1.89$ & $F=2.89$ & $F=2.9$ & $F=2.67$ & $F=4.64(p=0.032)$ & $F=4.45(p=0.035)$ & $F=5.23(p=0.023)$ \\
\hline Face & $F=3.32$ & $F=1.78$ & $F=1.78$ & $F=1.78$ & $F=2.98$ & $F=4.34(p=0.038)$ & $F=4.88(p=0.028)$ & $F=6.53(p=0.012)$ \\
\hline \multicolumn{9}{|c|}{ Control subjects $(n=11)$} \\
\hline Hand & $F=2.12$ & $F=1.56$ & $F=1.89$ & $F=1.29$ & $F=0.089$ & $F=4.16(p=0.045)$ & $F=4.05(p=0.048)$ & $F=4.14(p=0.045)$ \\
\hline Face & $F=2.45$ & $F=1.65$ & $F=1.43$ & $F=1.52$ & $F=0.28$ & $F=4(p=0.049)$ & $F=4.08(p=0.048)$ & $F=3.98(p=0.049)$ \\
\hline
\end{tabular}

The probabilities $p$ for significant comparisons are reported
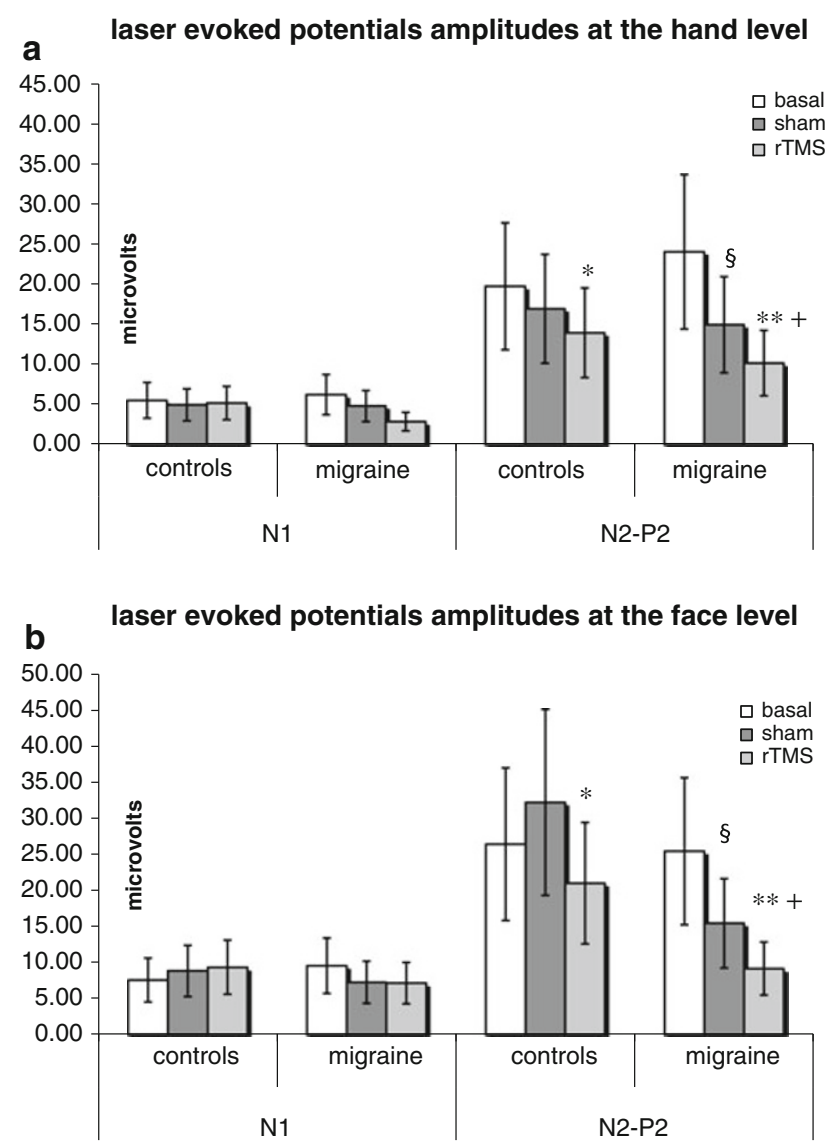

Fig. 2 Mean values and standard deviations of laser-evoked potentials amplitudes for the right hand (a) and the right supraorbital zone (b) in 12 controls and 13 migraine patients without aura. Subjects were evaluated in basal conditions, after sham TMS and after $5 \mathrm{~Hz}$ rTMS exposure at the left primary motor cortex. Results of multiple comparison Bonferroni tests of the single groups: basal versus rTMS: ${ }^{*} p<0.05, * * p<0.01$; basal versus sham: ${ }^{+} p<0.05$; sham versus rTMS: ${ }^{\S} p<0.05$

patients at the hand level. Although the small amplitude of N1 restricts its clinical use, the bipolar montage linking temporal and midline electrodes employed in this study generally renders it sizeable enough for detecting possible changes across different conditions [26], including changes in migraine patients [27]. EEG/MEG dipole analysis and intracortical recordings indicate that the $\mathrm{N} 1$ signal is mainly generated in the upper bank of the Sylvian fissure, encompassing the secondary somatosensory area (SII) and the posterior insula [28-31]. The present results may thus suggest that these cortical areas are not conditioned by the stimulation of the motor cortex, either in normal subjects or in migraine patients, in accordance with previous findings about the effects of voluntary movement on LEPs [13]. Furthermore, the N1 is less sensitive to attention and vigilance compared to the vertex complex [32], which may also explain the lack of a placebo effect in this component.

Regarding the point (3), at the hand and trigeminal levels, a significant amplitude reduction was observed in the vertex LEPs of the migraine patients during the sham session, suggesting that the TMS procedure has a strong placebo influence. In the control group, this placebo effect was present at the hand level but was not significant, and it was absent at the trigeminal level. Dipole modeling of scalp EEG signals and intracranial recordings suggests that the vertex LEP complex results from the simultaneous activity of several cortical generators, with major participation of the anterior cingulate cortex (ACC) and variable contribution from the insular and/or frontal operculum [28]. Its amplitude is especially sensitive to changes in attention and arousal levels [32] and also to the placebo effect, which mainly affects the P2 wave [33]. In our experiment, a separate analysis of the negative-positive components of the vertex complex did not show that N2 and P2 amplitudes behaved differently, suggesting that cortical areas devoted to both cognitive and emotive aspects of pain are inhibited by sham stimulation. We can assume that the placebo effect may prevail in patients compared to normal subjects, as patients will hope for a positive effect to alleviate their suffering. In addition, the placebo effect observed in the LEPs following stimulation of the trigeminal site in migraine patients and not in controls may be due to the different motivation and emotive involvement linked with the headache site. On the other hand, one could speculate that the inefficacy of a sham rTMS at the trigeminal zone in controls may be caused by the somatotopy of placebo effect, such as that suggested by Benedetti et al. [34]. The modulation of nociceptive 

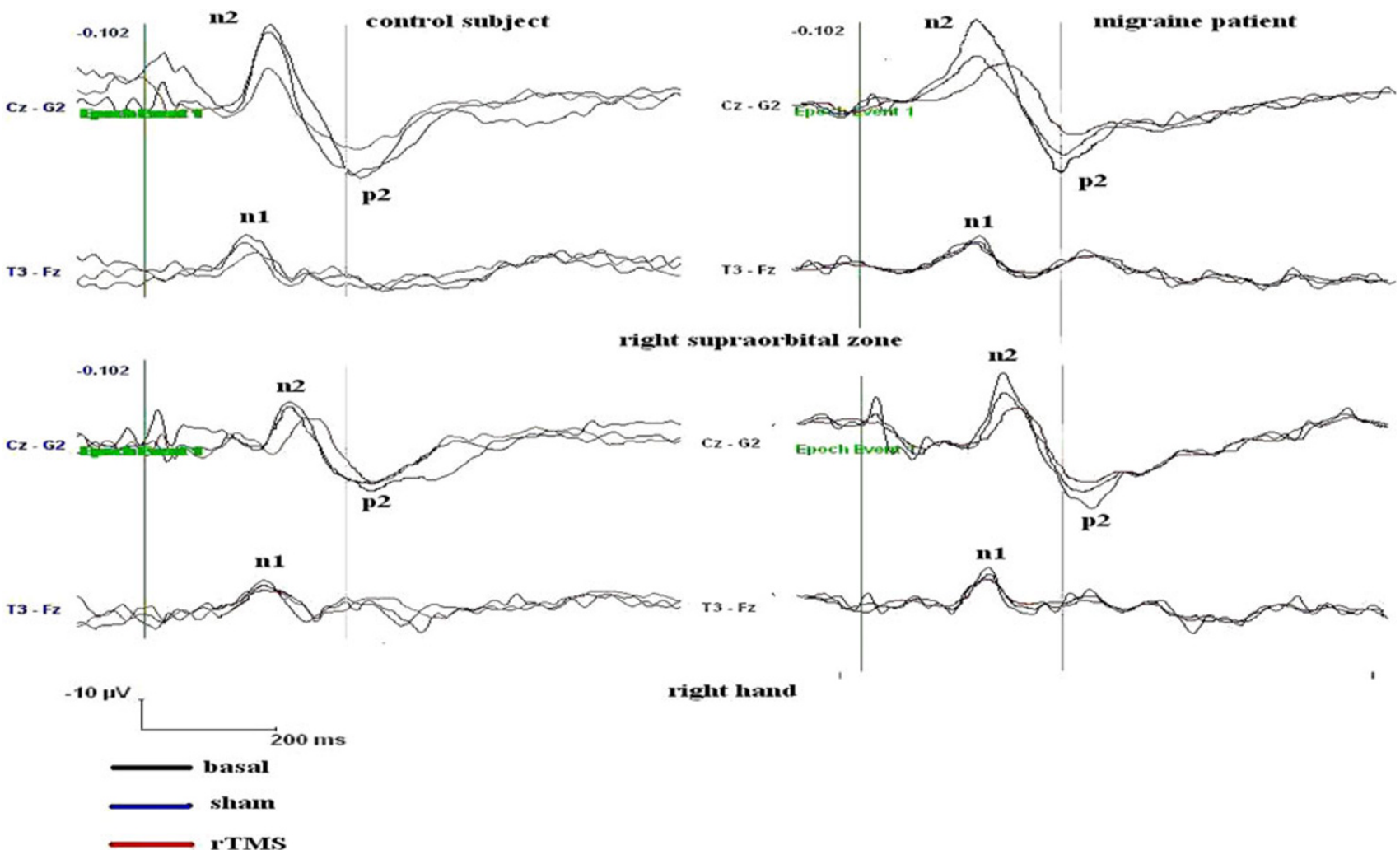

right hand

Fig. 3 Laser-evoked potentials of a representative control subject (female, 26 years old, right column) and a migraine patient without aura (female, 27 years old, left column) obtained following laser stimulation of the dorsum of the right supraorbital zone (upper panel)

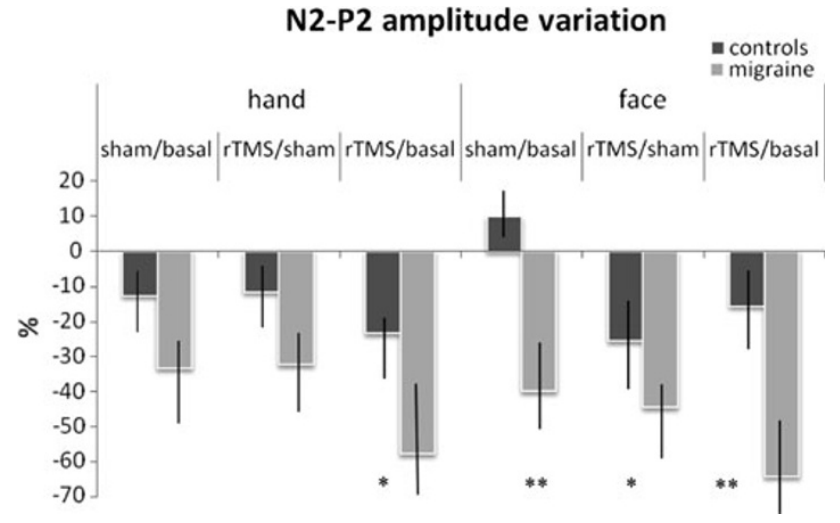

Fig. 4 Mean values and standard deviations of percent modification of N2-P2 amplitude between the basal, sham and rTMS conditions in migraine patients and controls. The results of Student's $t$ test, corrected for multiple comparisons, are shown: $* p<0.05$, $* * p<0.001$

processing has peculiar aspects in migraine patients, as it is less evident under distraction in cognitive tasks and is normally induced under distraction by affective images $[27,35]$. The sham TMS procedure induced a placebo effect on nociceptive processing in migraine patients, without an evident effect on subjective pain rating. This and the right hand (lower panel) by laser at an intensity of $8 \mathrm{~W}$ and a duration of $25 \mathrm{~ms}$ in basal conditions and after sham TMS and rTMS of the left primary motor cortex. Each trace represents the average of 20 artifact-free responses

apparent discrepancy was interpreted by Wager et al. [33] as a separate effect of placebo on early nociceptive processing, as shown by the P2 inhibition, and later processing of evaluation or cognitive judgment leading to the reduction of the pain rating. Similarly, Colloca et al. [36] observed a reduction in the N2-P2 amplitude in the absence of a reduction in the laser pain rating in a cohort of normal subjects subjected to the placebo effect by verbal suggestion.

Finally (point 4), we observed that HF TMS of the primary motor cortex induced a significant reduction in the vertex LEP amplitudes in migraine patients at both sites (hand and trigeminal), compared to basal and sham conditions. In contrast, HF TMS did not differ significantly from shams TMS in controls at the hand level. Compared to the basal sessions, a slight latency increase of about $30 \mathrm{~ms}$ was also detected in rTMS-treated migraine patients in the $\mathrm{N} 2$ and P2 components, but this difference did not reach statistical significance and needs to be confirmed using a larger data set. Our results suggest that the interaction between the motor and nociceptive cortex [15] predominantly involves the cortical areas subtending the vertex LEPs [28], in accordance with a functional relationship between M1 and ACC [37-39]. The effect of 
rTMS was similar for the negative and positive compounds of the vertex complex. Similarly, in a study by Le Pera et al. [13], the preparation of voluntary movement inhibited the vertex LEP amplitude, leaving the temporal N1 unchanged. In the study by Le Pera et al. [13], the inhibitory effect on the nociceptive system induced by physiological activation of the motor cortex involved only cerebral pain processing at the body site corresponding to the physiologically activated motor cortex. In our study, rTMS modulation of M1 realized on the area innervating the hand also reduced pain-related responses at the trigeminal level, suggesting that the inhibition of the nociceptive cortex is not dependent upon the somatotopic relationship between the stimulated M1 zone and the zone experiencing pain, as suggested by Lefaucheur et al. [6]. The effect of rTMS appeared to be more constant across the two evaluated sites in the migraine group than in the control group, which lacked significant late LEP reduction at the hand level following rTMS compared to the sham procedure. According to Curra et al. [40], a deficit in cortical inhibitory interneurons may affect the motor cortex in migraine patients, as well as in chronic neuropathic pain [14]. Restoration of GABAergic neurotransmission in the motor cortex induced by HF TMS may have an analgesic effect [14]. This hypothesis was recently confirmed by the same authors, who demonstrated a significant reduction in vertex LEPs and laser pain in a cohort of patients with chronic neuropathic pain [15]. The effects of HF TMS may be more evident when the motor cortex excitability is changed due to chronic pain [14]. In line with this theory, one might predict better results in chronic migraine patients, whose vertex LEPs are scarcely modified by cognitive distraction [27].

In summary, the state of the motor cortex in migraine patients could explain the more evident modulation of late LEPs induced by HF rTMS with respect to controls. Strong caution should be used in extending the validity of these findings to clinical practice. The study design was not suitable to evaluate clinical changes in our migraine patients, given that multiple consecutive sessions of rTMS are suggested to improve chronic pain [2] and specifically chronic migraine [17], and that the sham and rTMS procedures were applied in two consecutive days in the same patient. In addition, in migraine group, the LEPs were affected by a strong placebo effect, which is a well-known phenomenon in previous experiments exploring the efficacy of therapeutic procedures [41]. Moreover, the real rTMS exerted a considerable reduction of vertex LEPs in comparison with the sham, especially at trigeminal level. Presently, we can suggest that HF rTMS of motor cortex influenced nociceptive cortical responses better than other modalities of pain modulation $[18,27]$, also supported by a strong placebo effect, which potential advantage should not be ignored [42].

Conflict of interest None.

\section{References}

1. Barker AT, Jalinous R, Freeston IL (1985) Non-invasive stimulation of the human motor cortex. Lancet II:1106-1107

2. Fregni F, Freedman S, Pascual-Leone A (2007) Recent advances in the treatment of chronic pain with non-invasive brain stimulation techniques. Lancet Neurol 6(2):188-191

3. Chen R, Cros D, Curra A, Di Lazzaro V, Lefaucheur JP, Magistris MR, Mills K, Rösler KM, Triggs WJ, Ugawa Y, Ziemann U (2008) The clinical diagnostic utility of transcranial magnetic stimulation: report of an IFCN committee. Clin Neurophysiol 119(3):504-532

4. Pascual-Leone A, Bartres-Faz D, Keenan JP (1999) Transcranial magnetic stimulation: studying the brain-behaviour relationship by induction of 'virtual lesions'. Philos Trans R Soc Lond B Biol Sci 354:1229-1238

5. Migita K, Uozumi T, Arita K, Monden S (1995) Transcranial magnetic coil stimulation of motor cortex in patients with central pain. Neurosurgery 36:1037-1039

6. Lefaucheur JP, Drouot X, Menard-Lefaucheur I et al (2004) Neurogenic pain relief by repetitive transcranial magnetic cortical stimulation depends on the origin and the site of pain. J Neurol Neurosurg Psychiatry 75:612-616

7. Pleger B, Janssen F, Schwenkreis P, Volker B, Maier C, Tegenthoff M (2004) Repetitive transcranial magnetic stimulation of the motor cortex attenuates pain perception in complex regional pain syndrome type I. Neurosci Lett 356:87-90

8. Treede RD, Lorenz J, Baumgärtner U (2003) Clinical usefulness of laser-evoked potentials. Neurophysiol Clin 33(6):303-314

9. Tamura Y, Okabe S, Ohnishi T, Saito DN, Arai N, Mochio S, Inoue K, Ugawa Y (2004) Effects of 1-Hz repetitive transcranial magnetic stimulation on acute pain induced by capsaicin. Pain 107(1-2):107-115

10. Tamura Y, Hoshiyama M, Inui K, Nakata H, Qiu Y, Ugawa Y, Inoue K, Kakigi R (2004) Facilitation of A[delta]-fiber-mediated acute pain by repetitive transcranial magnetic stimulation. Neurology 22:2176-2181

11. Summers J, Johnson S, Pridmore S, Oberoi G (2004) Changes to cold detection and pain thresholds following low and high frequency transcranial magnetic stimulation of the motor cortex. Neurosci Lett 368(2):197-200

12. Yoo WK, Kim YH, Doh WS, Lee JH, Jung KI, Park DS, Park ES (2006) Dissociable modulating effect of repetitive transcranial magnetic stimulation on sensory and pain perception. Neuroreport 17(2):141-144

13. Le Pera D, Brancucci A, De Armas L, Del Percio C, Miliucci R, Babiloni C, Restuccia D, Rossini PM, Valeriani M (2007) Inhibitory effect of voluntary movement preparation on cutaneous heat pain and laser-evoked potentials. Eur J Neurosci 25(6):1900-1907

14. Lefaucher JP, Drouot X, Ménard-Lefaucher I, Keravel Y, Nguyen JP (2006) Motor cortex rTMS restores defective intracortical inhibition in chronic neuropathic pain. Neurology 67:1568-1574

15. Lefaucheur JP, Jarry G, Drouot X, Ménard-Lefaucheur I, Keravel Y, Nguyen JP (2010) Motor cortex rTMS reduces acute pain provoked by laser stimulation in patients with chronic neuropathic pain. Clin Neurophysiol (in press) 
16. O'Reardon JP, Fontecha JF, Cristancho MA, Newman S (2007) Unexpected reduction in migraine and psychogenic headaches following rTMS treatment for major depression: a report of two cases. CNS Spectr 12(12):921-925

17. Brighina F, Piazza A, Vitello G, Aloisio A, Palermo A, Daniele O, Fierro B (2004) rTMS of the prefrontal cortex in the treatment of chronic migraine: a pilot study. J Neurol Sci 227:67-71

18. de Tommaso M (2008) Laser-evoked potentials in primary headaches and cranial neuralgias. Expert Rev Neurother 8(9):1339-1345

19. Headache Classification Committee (2004) The international classification of headache disorders II. Cephalalgia 24:24-136

20. American Psychiatric Association (1994) Diagnostic and statistical manual of mental disorders (DSM-IV), 4th edn. American Psychiatric Association, Washington, DC

21. Valeriani M, Rambaud L, Mauguière F (1996) Scalp topography and dipolar source modelling of potentials evoked by $\mathrm{CO}_{2}$ laser stimulation of the hand. Electroencephalogr Clin Neurophysiol 100(4):343-353

22. Lefaucheur JP, Drouot X, Keravel Y, Nguyen JP (2001) Pain relief induced by repetitive transcranial magnetic stimulation of precentral cortex. Neuroreport 12:2963-2965

23. de Tommaso M, Losito L, Libro G, Guido M, Di Fruscolo O, Sardaro M, Sciruicchio V, Lamberti P, Livrea P (2005) Effects of symptomatic treatments on cutaneous hyperalgesia and laser evoked potentials during migraine attack. Cephalalgia 25(5):359-368

24. Bromm B, Treede RD (1991) Laser-evoked cerebral potentials in the assessment of cutaneous pain sensitivity in normal subjects and patients. Rev Neurol 147(10):625-643

25. Truini A, Panuccio G, Galeotti F, Maluccio MR, Sartucci F, Avoli M, Cruccu G (2010) Laser-evoked potentials as a tool for assessing the efficacy of antinociceptive drugs. Eur J Pain 14(2):222-225

26. Cruccu G, Aminoff MJ, Curio G, Guerit JM, Kakigi R, Mauguiere F, Rossini PM, Treede RD, Garcia-Larrea L (2008) Recommendations for the clinical use of somatosensory-evoked potentials. Clin Neurophysiol 119(8):1705-1719

27. de Tommaso M, Baumgartner U, Sardaro M, Difruscolo O, Serpino C, Treede RD (2008) Effects of distraction versus spatial discrimination on laser-evoked potentials in migraine. Headache 48(3):408-416

28. Garcia-Larrea L, Frot LM, Valeriani M (2003) Brain generators of laser-evoked potentials: from dipoles to functional significance. Neurophysiol Clin 33:279-292

29. Schlereth T, Baumgartner U, Magerl W, Stoeter P, Treede RD (2003) Left hemisphere dominance in early nociceptive processing in the human parasylvian cortex. Neuroimage $20: 441-454$

30. Kakigi R, Inui K, Tamura Y (2005) Electrophysiological studies on human pain perception. Clin Neurophysiol 116:743-763

31. Frot M, Magnin M, Mauguière F, Garcia-Larrea L (2007) Human SII and posterior insula differently encode thermal laser stimuli. Cereb Cortex 17:610-620

32. Lorenz J, Garcia-Larrea L (2003) Contribution of attentional, cognitive factors to laser evoked brain potentials. Neurophysiol Clin 33:293-301

33. Wager TD, Matre D, Casey KL (2006) Placebo effects in laserevoked pain potentials. Brain Behav Immun 20(3):219-230

34. Benedetti F, Arduino C, Amanzio M (1999) Somatotopic activation of opioid systems by target-directed expectations of analgesia. J Neurosci 19(9):3639-3648

35. de Tommaso M, Calabrese R, Vecchio E, De Vito Francesco V, Lancioni G, Livrea P (2009) Effects of affective pictures on pain sensitivity and cortical responses induced by laser stimuli in healthy subjects and migraine patients. Int $\mathrm{J}$ Psychophysiol 74(2): 139-148

36. Colloca L, Tinazzi M, Recchia S, Le Pera D, Fiaschi A, Benedetti F, Valeriani M (2008) Learning potentiates neurophysiological and behavioral placebo analgesic responses. Pain 15:306-314

37. Poreisz C, Csifcsák G, Antal A, Levold M, Hillers F, Paulus W (2008) Theta burst stimulation of the motor cortex reduces laserevoked pain perception. Neuroreport 19:193-196

38. Morecraft RJ, Van Hoesen GW (1992) Cingulate input to the primary and supplementary motor cortices in the rhesus monkey: evidence for somatotopy in area $24 \mathrm{c}$ and $23 \mathrm{c}$. J Comp Neurol 322:471-489

39. Siebner H, Peller M, Bartenstein P, Willoch F, Rossmeier C, Schwaiger M, Conrad B (2001) Activation of frontal premotor area during suprathreshold transcranial magnetic stimulation of the left primary sensorimotor cortex: a glucose metabolic PET study. Human Brain Mapp 12:157-167

40. Curra A, Pierelli F, Coppola G, Barbanti P, Buzzi MG, Galeotti F, Serrao M, Truini A, Casali C, Pauri F, Cruccu G (2007) Shortened cortical silent period in facial muscles of patients with migraine. Pain 132(1-2):124-131

41. Schwedt TJ, Hentz JG, Dodick DW (2007) Factors associated with the prophylactic effect of placebo injections in subjects enrolled in a study of botulinum toxin for migraine. Cephalalgia 27:528-534

42. Pollo A, Benedetti F (2009) The placebo response: neurobiological and clinical issues of neurological relevance. Prog Brain Res 175:283-289 